\title{
Academic detailing and adherence to guidelines for Group B streptococci prenatal screening: a randomized controlled trial
}

\author{
Jussara M Silva ${ }^{1 *}$, Airton T Stein ${ }^{2}$, Holger J Schünemann ${ }^{3}$, Ronaldo Bordin ${ }^{4}$, Ricardo Kuchenbecker ${ }^{5}$ \\ and Maria de Lourdes Drachler ${ }^{6}$
}

\begin{abstract}
Background: Clinical practice guidelines (CPGs) recommend universal prenatal screening for Group B Streptococcus (GBS) to identify candidates for intrapartum antibiotic prophylaxis to prevent early onset neonatal GBS infection. Interventions to promote physician adherence to these guidelines are imperative. This study examined the effectiveness of academic detailing (AD) of obstetricians, compared with CPG mailshot and no intervention, on the screening of pregnant women for GBS.

Methods: A randomized controlled clinical trial was conducted in the medical cooperative of Porto Alegre, Brazil. All obstetricians who assisted in a delivery covered by private health insurance managed by the cooperative in the 3 months preceding the study ( $n=241)$ were invited to participate. The obstetricians were randomized to three groups: direct mail (DM, $n=76), A D(n=76)$ and control (C, $n=89$, no intervention). Those in the DM group were sent guidelines on GBS. The AD group received the guidelines and an educational visit detailing the guidelines, which was conducted by a trained physician. Data on obstetrician age, gender, time since graduation, whether patients received GBS screening during pregnancy, and obstetricians who requested screening were collected for all participant obstetricians for 3 months before and after the intervention, using database from the private health insurance information system.

Results: Three months post-intervention, the data showed that the proportion of pregnant women screened for GBS was higher in the AD group (25.4\%) than in the DM (15.9\%) and $C(17.7 \%)$ groups $(P=0.023)$. Similar results emerged when the three groups were taken as a cluster (pregnant women and their obstetricians), but the difference was not statistically significant (Poisson regression, $P=0.108$ ). Additionally, when vaginal deliveries were analyzed separately, the proportion screened was higher in the AD group (75\%) than in the DM group (41.9\%) and the C group (30.4\%) (chi-square, $P<0.001$ ).
\end{abstract} Conclusions: The results suggest that $A D$ increased the prevalence of GBS screening in pregnant women in this population. Keywords: Guidelines, Physicians, Pregnancy, Screening, Streptococci

\section{Background}

Group B Streptococcus (GBS) infection is the most common bacterial infection transmitted vertically from mother to child during labor and delivery. The neonatal infection is a leading cause of neonatal morbidity and mortality and can severely affect the quality of life of the child in the short and long term. Efforts are needed internationally to prevent neonatal GBS infections.

\footnotetext{
* Correspondence: jussaramunareto@gmail.com

${ }^{1}$ Department of Epidemiology, Federal University of Rio Grande do Sul, Porto Alegre, Brazil

Full list of author information is available at the end of the article
}

Medical guidelines on prenatal care strongly recommend GBS screening of the vagina and anus between 35 and 37 weeks of gestation and intrapartum antibiotic prophylaxis of colonized women to prevent neonatal infection $[1,2]$. Despite their success as a strategy to prevent perinatal GBS disease, these recommendations have mostly been applied in developed countries only [3]. In Brazil, a prevalence of maternal colonization by GBS of between 15 and $25 \%$ suggests that universal screening is likely to be cost-effective in this country $[4,5]$, and GBS screening is recommended by the Brazilian Medical Guidelines on prenatal care [2]. Interventions potentially

\section{Biomed Central}

(c) 2013 Silva et al.; licensee BioMed Central Ltd. This is an Open Access article distributed under the terms of the Creative Commons Attribution License (http://creativecommons.org/licenses/by/2.0), which permits unrestricted use, distribution, and reproduction in any medium, provided the original work is properly cited. 
effective in promoting the adherence of obstetricians to these guidelines are lacking in this country.

Internationally, the implementation of medical guidelines is a challenge that usually takes many years $[6,7]$. Passive continuing medical education, or traditional education, appears to be of limited effect in guideline implementation. Studies have shown that educating physicians in their office is a promising strategy for changing medical practice $[8,9]$, mainly through academic detailing (AD), an intervention that combines interactive, one-on-one communication conducted by trained healthcare professionals-typically pharmacists, physicians or nurses-with evidence-based, noncommercial information. AD has been successfully applied to increase the adherence of health professionals to guidelines for screening $[10,11]$ and to decrease inappropriate use of medicines [9]. It involves face-to-face education, with additional elements such as educational materials, educational meetings, or audit and feedback [12].

Some studies have assessed the effectiveness of $A D$ in the implementation of guidelines in obstetrics [13], with mixed effects [13] and favorable results [14]. Multifaceted behavioral interventions, including AD visits, appeared to increase the prophylactic use of oxytocin during the third stage of labor and reduced the likelihood of episiotomy in one study [15].

No research has investigated the effect of AD in promoting GBS screening in the private or public sector in Brazil. This study examined the effectiveness of $A D$, conducted through an educational visit by a trained physician to obstetricians, in promoting screening for vaginal and rectal GBS colonization.

\section{Methods}

A randomized controlled clinical trial of interventions to promote prenatal screening for GBS was conducted. The participant service was a major private health insurance company managed by a medical cooperative in Porto Alegre, South Brazil [16]. All obstetricians $(n=241)$ who had provided outpatient prenatal care and assisted in at least one delivery (vaginal or cesarean section) covered by this health insurance in the 3 months before the study (April to June, 2008) were invited to take part. The same doctors were involved in prenatal care. The participant obstetricians were allocated randomly into three groups: direct mail (DM, $n=76), \mathrm{AD}(n=76)$ and control $(C, n=89)$. A list of random numbers generated by the Statistical Package for the Social Sciences (SPSS Inc., Chicago, IL, USA) was used to allocate the obstetricians.

The DM intervention comprised printed guidelines on antenatal GBS screening sent by post to the obstetrician's private office in July, 2008. The AD intervention included these guidelines and a 30-minute face-to-face educationoriented interview on antenatal GBS screening based on the national guidelines for antenatal care [2]. This was conducted by a trained physician and took place in the private offices of the participating obstetricians, in July and August, 2008. No intervention was provided to the $C$ group.

The electronic database of the participating organization was used to gather the following information about the obstetricians and their performance of antenatal care and deliveries both 3 months pre-intervention (April to June, 2008) and 3 months post-intervention: obstetrician age, gender, year of graduation in medicine, number of years of antenatal clinical practice in the medical cooperative, number of births assisted, and the frequency of GBS screening requested.

Multivariable Poisson regression analysis was used to investigate the effect of the intervention on the proportion of pregnant women tested for GBS, controlling for potential confounding factors (physician age and sex, time since graduation in medicine and number of years of antenatal clinical practice in the medical cooperative). A $P$ value of 0.05 or less was considered statistically significant.

The study was approved by the Ethics Committee of Federal University of Rio Grande do Sul, protocol number 2007792 .

\section{Results}

There were 908 deliveries during the baseline assessment period. There was no evidence of a difference between the three groups of obstetricians (AD, DM and C) in relation to the baseline variables: age, gender, number of years since graduation in medicine, number of years of antenatal clinical practice in the medical cooperative, proportion of obstetricians requesting a GBS screening, and proportion of pregnant women tested for GBS (15.8\%, $17.0 \%$ and $21.4 \%$, respectively) (Table 1 ).

There were 849 deliveries during the 3 -month postintervention period. In this time, 58, 67 and 78 obstetricians in the $\mathrm{AD}, \mathrm{DM}$ and $\mathrm{C}$ groups, respectively, performed at least one delivery. The other 38 obstetricians did not assist with a delivery in this period.

In the post-intervention period, there was evidence of a difference between the three groups in the proportion of pregnant women tested for GBS (chi-square, $P=0.023$ ); the proportion was higher for the AD group (25.4\%) than for the DM group (15.9\%) and the C group (17.2\%) (Table 2).

Additionally, when the three groups were considered as a cluster (pregnant women and their obstetricians), the frequency of GBS screening was higher for the AD group than for the $\mathrm{C}$ group, but this difference was not statistically significant (Poisson regression, $P=0.108$ ) (Table 3).

Similarly, when vaginal deliveries were analyzed separately, the proportion of GBS screening was higher in the 
Table 1 Sociodemographic data of obstetricians and details of their practice before the intervention $(n=241)$

\begin{tabular}{|c|c|c|c|c|}
\hline & $A D^{a}$ & DM & C & $p$-value \\
\hline & $\mathrm{N}=76$ & $\mathrm{~N}=76$ & $\mathrm{~N}=89$ & \\
\hline Age (in years), mean ${ }^{b}$ & $45.6 \pm 8.6$ & $45.7 \pm 8.0$ & $46.1 \pm 8.0$ & $p=0.90$ \\
\hline Female, $\%^{c}$ & 75.0 & 63.2 & 62.9 & $p=0.251$ \\
\hline Time elapsed since graduation in medicine (in years), mean ${ }^{b}$ & $21.2 \pm 8.5$ & $21.2 \pm 7.7$ & $21.1 \pm 8.1$ & $p=0.99$ \\
\hline Time in healthcare plan (in years), mean ${ }^{b}$ & $15.5 \pm 8.1$ & $15.7 \pm 6.6$ & $16.3 \pm 7.2$ & $p=0.84$ \\
\hline Obstetricians who had requested GBS screening pre-intervention ${ }^{d}, \%^{c}$ & 31.6 & 26.3 & 37.1 & $p=0.33$ \\
\hline Pregnant women tested for GBS pre-intervention ${ }^{d}, n$ and (\%) ${ }^{c}$ & $38 / 240(15.8)$ & $59 / 346(17.05)$ & $69 / 322(21.42)$ & $p=0.19$ \\
\hline
\end{tabular}

a AD: Academic detailing; DM: direct mail; C: control group; Data are presented as ${ }^{b}$ mean \pm standard deviation, ${ }^{c}$ numbers (percentage); ${ }^{d}$ Group B streptococcus (GBS) screening in the 3 months before the intervention.

AD group (75\%) than in the DM group (41.9\%) and the C group (30.4\%) (chi-square, $P<0.001)$ (Table 4).

\section{Discussion}

This paper reports a randomized controlled clinical trial of an intervention implemented in the context of usual prenatal outpatient care. The trial showed that pregnant women cared for by obstetricians who received an ADbased intervention were more likely to be screened for GBS than were those cared for by obstetricians who received printed guidelines only or no intervention. Similar results emerged when the three groups were taken as a cluster (pregnant women and their obstetricians), but the difference was not statistically significant, maybe because of low study power. The study was based on obstetrician members of a medical cooperative and the number of obstetricians and patients was relatively small. There was also loss of participant obstetricians on intention-to-treat analysis because 38 obstetricians did not conduct deliveries during the study period.

The AD intervention was associated with a significant increase of $9.5 \%$ in the frequency of prenatal GBS screenings compared with the passive printed material distribution or the no intervention scenario. This modest result for $\mathrm{AD}$ is in line with similar studies on guideline implementation [17].

In the present study, when vaginal deliveries were analyzed separately, the proportion of screening was higher in the $\mathrm{AD}$ group (75\%) than in the DM group (41.9\%) and the $C$ group (30.4\%). These results suggest that the $\mathrm{AD}$ intervention was particularly relevant in women who had a vaginal delivery, for which prenatal GBS screening is the most useful in preventing neonatal infection.

The trial was conducted with obstetricians who had assisted a delivery paid for by the medical cooperative in the 3 months before the intervention, regardless of whether the obstetrician had requested GBS screening. The sample size was insufficient to analyze the effect of the intervention separately for obstetricians who had and had not previously requested screening. Because some studies have shown no impact of untargeted outreach visits $[18,19]$, further studies should investigate the effect of AD on GBS screening separately for these two groups. Outreach visits may also face barriers in the form of resistance to change [20], which should be assessed in future studies. Factors that most discourage the use of $\mathrm{AD}$ are time spent in the office for continuing medical education, physicians' perception of wasting working time in the office to receive AD and continuing medical education provided by a non-physician [21]. The attitudes of the obstetricians were not assessed in the present study.

Another limitation of this trial is the relatively short follow-up of 3 months, which may have led to overestimation of the observed benefit of the intervention. Additionally, there is a possibility of contamination of the DM and $\mathrm{C}$ groups, but underestimation of the effect of

Table 2 Requests for and performance of GBS testing in pregnant women for 3 months post-intervention

\begin{tabular}{|c|c|c|c|c|}
\hline & $A D^{a}$ & DM & C & Chi-square $p$-value \\
\hline & $\mathrm{N}=76$ & $\mathrm{~N}=76$ & $\mathrm{~N}=89$ & \\
\hline \multicolumn{5}{|l|}{ Obstetricians } \\
\hline Who requested $\mathrm{GBS}^{\mathrm{b}}$ screening post intervention ${ }^{c}$ & $28(36.8)$ & $21(27.6)$ & $32(30.0)$ & 0.412 \\
\hline \multicolumn{5}{|l|}{ Pregnant } \\
\hline Proportion of pregnant women tested for GBS post intervention ${ }^{\mathrm{d}}$ & $52 / 205(25.36)$ & $45 / 283(15.90)$ & $64 / 361(17.72)$ & 0.023 \\
\hline
\end{tabular}

${ }^{a}$ AD: Academic detailing; DM: direct mail; C: control group; ${ }^{\mathrm{b}}$ Group B streptococcus- one or more cultures; ${ }^{\mathrm{c}}$ Data are presented as numbers (percentage);

${ }^{d}$ Number of pregnant women screened for GBS divided by the number of women who gave birth in the period. 
Table 3 Comparison between the intervention groups and control group on application and performance of GBS testing in pregnant women, considering the cluster "pregnant women and their obstetricians"

\begin{tabular}{llll}
\hline Requested culture GBS & Prevalence rate & $\mathbf{9 5 \%} \mathrm{Cl}^{\mathbf{a}}$ & $\boldsymbol{p}$ \\
\hline $\mathbf{A D}^{\mathbf{b}}$ group & 1.43 & $0.92-2.21$ & 0.108 \\
$\mathbf{D M}^{\mathbf{b}}$ group & 0.89 & $0.53-1.51$ & 0.683 \\
$\mathbf{C}^{\mathbf{b}}$ & Reference & & \\
\hline
\end{tabular}

${ }^{a}$ confidence interval; ${ }^{\mathrm{b}}$ AD: Academic detailing; DM: direct mail; C: control group.

$\mathrm{AD}$ is unlikely because the obstetricians worked in private medical outpatient practices and had relatively little interaction with each other.

In this trial, the educational visits were conducted by a trained physician, in line with a previous study that showed that visits made by peers tend to be more effective for behaviors related to collaboration with others and practice organization, compared with interventions conducted by non-peers [22]. However, interventions provided to obstetricians by other health professionals, such as nurses, should be examined in other studies.

This study has the potential to contribute to best practice, showing that a brief intervention based on $A D$ in medical practice may have a significant impact in increasing the number of patients screened for GBS. This study is also relevant to obstetric practice in middle income economies such as Brazil where a significant proportion of the population receives care paid through private health insurance.

Several factors may prevent obstetricians following prenatal screening policy. Among these is the fact that there is a high rate of cesarean section in private clinics in Brazil (e.g. 83.3\% in the present study), which may prompt obstetricians to consider the promotion of prenatal GSB screening unnecessary, especially for women already scheduled to undergo elective cesarean section. While the World Health Organization recommends a maximum of $15 \%$ cesarean sections among total births, Brazil has one of the highest cesarean section rates in the world, with a national average of $43 \%$, reaching $80 \%$

Table 4 Effect of interventions on requests for and performance of culture for Group B streptococci in women with vaginal deliveries

\begin{tabular}{llll}
\hline Groups & $\begin{array}{l}\mathbf{N}^{\mathbf{o}} \text { Vaginal } \\
\text { dēliveries }\end{array}$ & $\begin{array}{l}\mathbf{N}^{\mathbf{0}} \mathrm{GBS}^{\mathbf{a}} \\
\mathbf{s c r e e n i n g}\end{array}$ & $\begin{array}{l}\% \mathrm{GBS}^{\mathbf{a}} \\
\text { screening }\end{array}$ \\
\hline $\begin{array}{l}\text { Academic } \\
\text { detailing }\end{array}$ & 32 & 24 & 75 \\
Direct mail & 43 & 18 & 41.9 \\
Control & 56 & 17 & 30.4 \\
Total & $\mathbf{1 3 1}$ & $\mathbf{5 9}$ & $\mathbf{4 5 . 0}$ \\
\hline
\end{tabular}

${ }^{\mathrm{a} G r o u p ~ B ~ s t r e p t o c o c c i ; ~ C h i-s q u a r e ~ 2 d F, ~} p<0.001$. in the private healthcare setting [23]. Although this is strongly related to higher social class, the main determinant of the elevated rate of cesarean section is delivery in a private maternity unit $[24,25]$, as the sample studied here shows. The main reasons given by obstetricians are the convenience of programmed intervention for the obstetrician, uncertainty regarding the possibility of hypoxia or fetal trauma, and lack of preparation of the woman for the birth [26,27].

The high cesarean section rate in the organization studied here may have played a role in the observed low impact of $\mathrm{AD}$, as suggested by the finding that $\mathrm{AD}$ had a greater effect when women who underwent vaginal delivery were analyzed separately. The obstetrician's decision to perform a cesarean section may have influenced his or her decision on whether to perform GBS screening because the aim of identifying women harboring GBS is to prevent neonatal colonization during vaginal delivery and not during cesarean section.

From the perspective of clinical practice in low resource settings, there may be gaps between the scientific evidence for an intervention and its adoption in clinical practice [28], including a lack of financial and nonfinancial resources to implement changes in healthcare. The low remuneration of medical care is likely to contribute to the high rate of caesarean sections in Brazil, especially in the private health sector, whereas vaginal delivery requires more working hours and lower remuneration proportionally. Financial incentives may be effective in changing healthcare professional practice [29]. Although GBS testing is an easy and affordable screening method, the adoption of this practice is likely to be affected by the use of elective cesarean section. Further studies are needed to establish whether opting for a cesarean is a barrier to GBS screening. Advances in behavioral economics are driving efforts to use material or financial incentives to overcome economic obstacles or a lack of effective motivation, and recipients are incentivized to engage in health-related behaviors or practices with which they are already familiar and that they regard as beneficial or worthwhile.

\section{Conclusions}

In conclusion, a benefit was achieved by the AD intervention in this study, in that more pregnant women were screened for GBS. Further longer term studies are needed before the benefits of $\mathrm{AD}$ in promoting the adoption of evidence-based guidelines on prenatal care are fully understood.

\section{Consent}

Written informed consent was obtained from all participant obstetricians for publication of this report. 


\section{Competing interests}

JM Silva is an Advisor to the Cooperative, a member of the Commission for Support and Guidance for implementation of Clinical Guidelines. The authors declare that they have no competing interests.

\section{Authors' contributions}

JMS - designed the overall study and conducted the analysis and drafted the manuscript. ATS - designed the overall study conducted the analysis and drafted the manuscript. HJS - conducted the analysis and drafted the manuscript. RB - conducted the analysis and drafted the manuscript. RK - conducted the analysis and drafted the manuscript. MLD - conducted the analysis and drafted the manuscript. All authors read and approved the final manuscript.

\section{Acknowledgements}

The author thanks Dr. Alice Zelmanowicz for her assistance in the elaboration of this study, the Medical Cooperative for granting permission to carry out the study and evaluating the office visits, and to UFRGS for providing the knowledge for research development.

\section{Author details}

'Department of Epidemiology, Federal University of Rio Grande do Sul, Porto Alegre, Brazil. ${ }^{2}$ Department of Epidemiology, Federal University of Health Sciences (UFCSPA) and Ulbra, Porto Alegre, Brazil. ${ }^{3}$ Clinical Epidemiology \& Biostatistics Department and Department of Medicine, McMaster University, Hamilton, Canada. ${ }^{4}$ Department of Epidemiology, School of Medicine, Federal University of Rio Grande do Sul, Porto Alegre, Brazil. ${ }^{5}$ School of Medicine, Federal University of Rio Grande do Sul, Hospital das Clinicas of Porto Alegre, Porto Alegre, Brazil. ${ }^{6}$ Postgraduate Programme in Collective Health, School of Nursing, Federal University of Rio Grande do Sul, Porto Alegre, Brazil.

Received: 3 May 2012 Accepted: 28 February 2013

Published: 19 March 2013

\section{References}

1. Centers for Disease Control and Prevention: Laboratory practices for prenatal group B streptococcal screening: 2003. Morb Mortal Wkly Rep 2004, 53:506-9.

2. Melo VH, Pires do Rio SM: Assistência Pré-natal. [http://www. projetodiretrizes.org.br/5_volume/02-AssistPre.pdf]

3. Edmond KM, Kortsalioudaki C, Scott S, et al: Group B streptococcal disease in infants aged younger than 3 months: systematic review and meta-analysis. Lancet 2012, 379(9815):547-56. doi:10.1016/S0140-6736(11)61651-6. Epub 2012 $\operatorname{Jan} 4$

4. Beraldo C, Brito ASJ, Saridakis HO, Matsuo T: Prevalência de colonização vaginal e anorretal por estreptococo do grupo $B$ em gestantes de terceiro trimestre. Rev Bras Ginecol Obstet 2004, 26(7):543-9.

5. Strickland DM, Yeomans ER, Hankins GD: Cost-effectiveness of intrapartum screening and treatment for maternal group B streptococci colonization. Am J Obstet Gynecol 1990, 163(1 Pt 1):4-8.

6. Majumdar SR, McAlister FA, Furberg CD: From knowledge to practice in chronic cardiovascular disease: a long and winding road. J Am Coll Cardiol 2004, 43:1738-1742

7. Balas EA, Boren SA: Yearbook of Medical Informatics: Managing Clinical Knowledge for Health Care Improvement. Stuttgart, Germany: Schattauer Verlagsgesellschaft mbH; 2000.

8. O'Brien MA, Rogers S, Jamtvedt G, Oxman AD, Odgaard-Jensen J, Kristoffersen DT, Forsetlund L, Bainbridge D, Freemantle N, Davis DA, et al.: Educational outreach visits: effects on professional practice and health care outcomes. Cochrane Database Syst Rev 2007, 4:CD000409.

9. David A, Davis MD, Taylor-Vaisey A: Translating guidelines into practice. A systematic review of theoretic concepts, practical experience and research evidence in the adoption of clinical practice guidelines. CMAJ 1997, 157(4):408-16.

10. Gorin SS, Ashford AR, Lantigua R, Hossain A, Desai M, Troxel A, Gemson D: Effectiveness of academic detailing on breast cancer screening among primary care physicians in an underserved community. J Am Board Fam Med 2006, 19(2):110-21.

11. Lobo CM, Frijling BD, Hulscher ME, Bernsen RM, Braspenning JC, Grol RP, Prins A, van der Wouden JC: Improving quality of organizing cardiovascular preventive care in general practice by outreach visitors: a randomized controlled trial. Prev Med 2002, 35(5):422-9.

12. Grimshaw JM, Thomas RE, MacLennan G, Fraser C, Ramsay CR, Vale L, Whitty P, Eccles MP, Matowe L, Shirran E, Wensing M, Dijkstra R, Donaldson C: Effectiveness and efficiency of guideline dissemination and implementation strategies. Health Technol Assess 2004, 8(6):1-72.

13. Chaillet N, Dubé E, Dugas M, Audibert F, Tourigny C, Fraser WD, Dumont A: Evidence-based strategies for implementing guidelines in obstetrics: a systematic review. Obstet Gynecol 2006, 108(5):1234-45.

14. Wyatt JC, Paterson-Brown S, Johanson R, Altman DG, Bradburn MJ, Fisk NM: Randomised trial of educational visits to enhance use of systematic reviews in 25 obstetric units. BMJ 1998, 317:1041-6.

15. Althabe F, Buekens P, Bergel E, Belizán JM, Campbell MK, Moss N, Hartwell T, Wright LL: A behavioral intervention to improve obstetrical care. $N$ Engl J Med 2008, 358(18):1929-40.

16. Duarte CMR: UNIMED: history and characteristics of a Brazilian medical cooperative. Cad. Saúde Pública, Rio de Janeiro 2001, 17(4):999-1008.

17. Oxman AD, Thomson MA, Davis DA, Haynes RB: No magic bullets: a systematic review of 102 trials of interventions to improve professional practice. CMAJ 1995, 153(10):1423-31.

18. Hall L, Eccles M, Barton R, Steen N, Campbell M: Is untargeted outreach visiting in primary care effective? A pragmatic randomized controlled trial. J Pub Health Med 2001, 23(2):109-113.

19. Freemantle N, Nazareth I, Eccles M, Wood J, Haines A, Trialists EOREBOR: A randomised trial of the effect of educational outreach by community pharmacists on prescribing in UK general practice. Br J Gen Pract 2002, 52(477):290-95.

20. Cabana MD, Rand CS, Powe NR, Wu AW, Wilson MH, Abboud PA, Rubin HR: Why Don't physicians follow clinical practice guidelines? A framework for improvement. JAMA 1999, 282(15):1458-1465.

21. Allen M, Ferrier S, O'Connor N, Fleming I: Family physicians' perceptions of academic detailing: a quantitative and qualitative study. BMC Med EduC 2007, 7:36

22. van den Hombergh $\mathrm{P}$, Grol R, van den Hoogen HJ, van den Bosch WJ: Practice visits as a tool in quality improvement: mutual visits and feedback by peers compared with visits and feedback by non physician observers. Qual Health Care 1999, 8(3):161-6.

23. Patah LEM, Malik AM: Models of childbirth care and cesarean rates in different countries. Rev Saúde Pública 2011, 45(1):185-194

24. Salvador J, Cano-Serral G, Rodríguez-Sanz M, Lladonosa A, Borrell C: Short report: Inequalities in caesarean section: influence of the type of maternity care and social class in an area with a national health system. J Epidemiol Community Health 2009, 63:259-26.

25. Gomes UA, Silva AA, Bettiol H, Barbieri MA: Risk factors for the increasing caesarean section rate in Southeast Brazil: a comparison of two birth cohorts, 1978-1979 and 1994. Int J Epidemiol 1999, 28(4):687-94.

26. Béhague DP, Victora CG, Barros FC: Consumer demand for caesarean sections in Brazil: informed decision making, patient choice, or social inequality? A population based birth cohort study linking ethnographic and epidemiological methods. BMJ 2002, 324(7343):942-5.

27. Joseph KS, Young DC, Dodds L, O'Connell CM, Allen VM, Chandra S, et al: Changes in maternal characteristics and obstetric practice and recent increases in primary cesarean delivery. Obstet Gynecol 2003, 102(4):791-800.

28. Martis R, Ho JJ, Crowther CA: Survey of knowledge and perception on the access to evidence-based practice and clinical practice change among maternal and infant health practitioners in South East Asia. BMC Pregnancy Childbirth 2008, 8:34.

29. Flodgren G, Eccles MP, Shepperd S, Scott A, Parmelli E, Beyer FR: An overview of reviews evaluating the effectiveness of financial incentives in changing healthcare professional behaviours and patient outcomes. Cochrane Database Syst Rev 2011, 6(7):CD009255. doi:10.1002/14651858. CD009255

\section{doi:10.1186/1471-2393-13-68}

Cite this article as: Silva et al:: Academic detailing and adherence to guidelines for Group B streptococci prenatal screening: a randomized controlled trial. BMC Pregnancy and Childbirth 2013 13:68. 